\section{Preparation of sections from bone marrow aspirates and cellular exudates using soluble plastic centrifuge tubes}

\section{S. W. A. KUPER From Brompton Hospital, London}

Sections are frequently helpful in the examination of bone marrow aspirates and of the cellular elements of body fluids. The technical difficulties involved have resulted in the elaboration of several time-consuming methods, often needing considerable skill and experience (Berman, 1953; Raman, 1955).

During the last few years, the manufacture of chloroform-soluble plastic tubes has made available a simple method in which the tube itself is dissolved after centrifugation, liberating the undisturbed 'button' of cells. These buttons have proved easy to handle, and satisfactory sections have been obtained.

\section{METHOD}

Marrow, 0.2 to $0.3 \mathrm{ml}$, is aspirated in the usual way, and

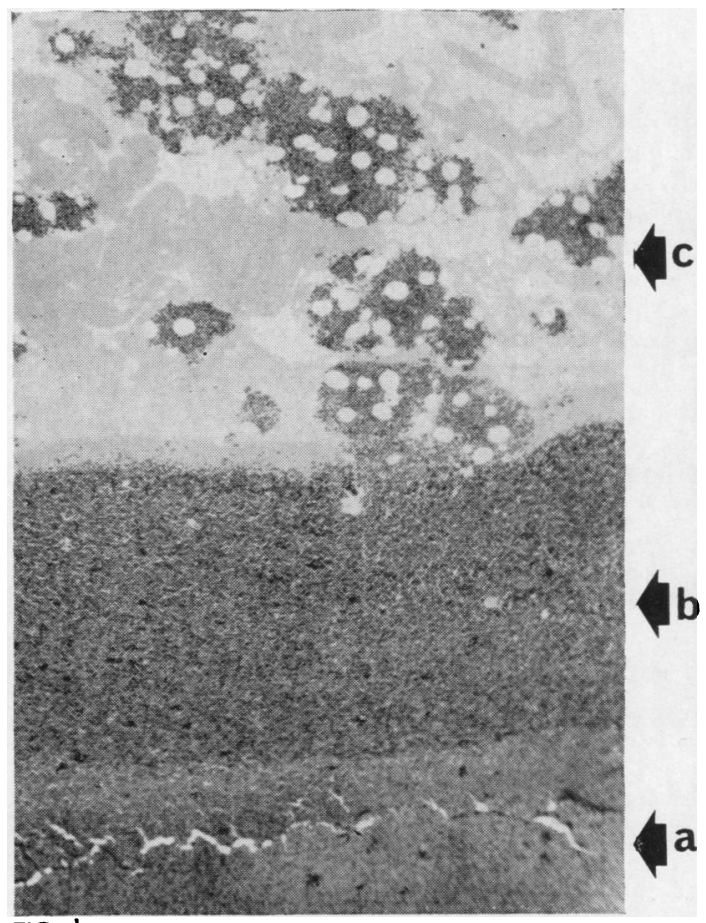

placed in a disposable crystal polystyrene precipitin tube $(6.35 \mathrm{~mm} . \times 38.1 \mathrm{~mm} \text {., external dimensions })^{1}$, containing a small crystal of E.D.T.A. (approximately $5 \mathrm{mg}$. of the potassium salt). It is carefully agitated to ensure thorough mixing. After centrifugation at 2,000 to 3,000 r.p.m. for 15 minutes, most of the supernatant plasma is pipetted off and replaced with fixative (equal volumes of absolute ethanol and $15 \%$ formalin). This is removed after a few minutes and replaced with fresh fixative which is allowed to act overnight. (The cellular deposits from serous effusions, etc., are frequently very small and a more convenient fixative for these is Bouin's fluid which stains the deposits yellow and makes them more easily visible during processing.) Next morning the tube and its contents are placed in a small bottle of warm chloroform, which is put in a water bath at 37 or $56^{\circ} \mathrm{C}$. (whichever is available) and gently agitated at intervals until the tube falls away releasing the cell button. The latter maintains its form if handled gently and may be dehydrated and embedded in paraffin wax as though it were a piece of tissue. Sections are cut longitudinally through the button, to reveal the different strata which compose it. At the base lie the heaviest cells, the erythrocytes. Above them is a zone of leucocytes and isolated marrow cells, and the upper layer consists of marrow fragments and megakaryocytes (Fig. 1). When the material contains no actual

1Obtainable from Luckham Ltd., London, S.W.20. (This firm has recently produced sheets of PVC with conical indentations. These can be cut out with scissors and used for centrifugation, and they are very readily soluble in chloroform.)

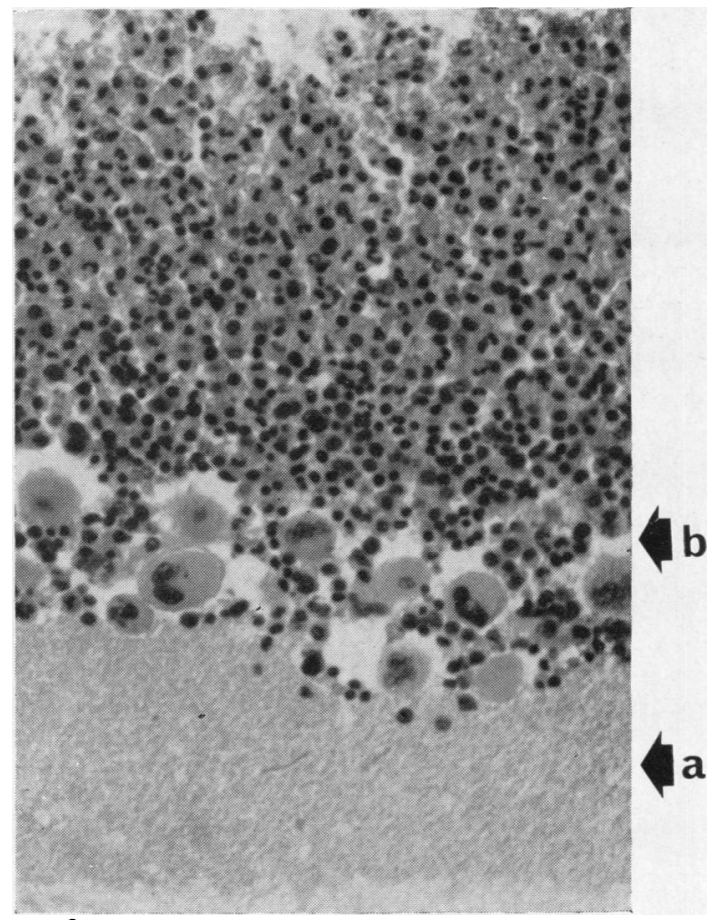

FIG. 2 


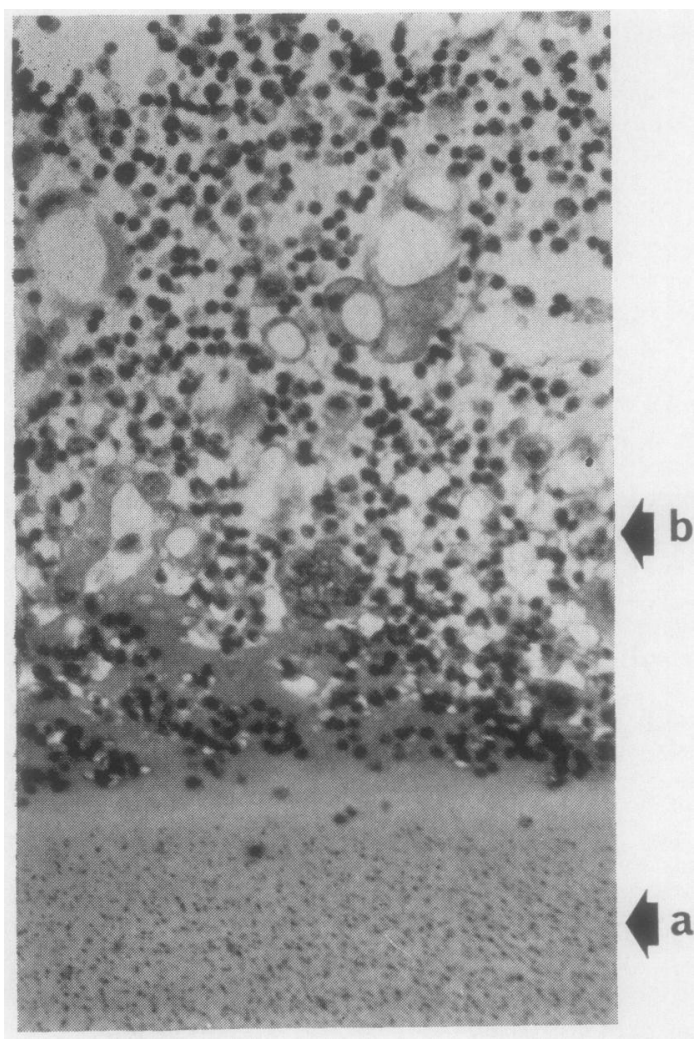

FIG. 3. Section from a pleural aspirate containing neoplastic cells.

fragments, any marrow elements present tend to accumulate in a definite layer above the packed erythrocytes (Fig. 2).

When processing serous effusions or liquified sputum, the volumes involved may be very large and the numbers of cells small. In such cases, preliminary concentration of cell suspensions is carried out by centrifugation in large tubes, the concentrated cell suspension being transferred to the plastic tubes for packing and fixation. On section, any red cells present are seen to lie at the bottom of the button. Leucocytes, mesothelium, and tumour cells lie in the upper layers (Fig. 3).

I am greatly indebted to Mr. M. C. Sale and Mr. G. B. Snell for generous and enthusiastic technical assistance and to Mr. K. Moreman of the Chester Beatty Research Institute, for the photographs.

\section{REFERENCES}

Berman, L. (1953). Amer. J. clin. Path., 23, 385.

Raman, K. (1955). J. clin. Path., 8, 265.

Received for publication 13 May 1964.

\section{Method for the detection and measurement of antibodies to streptokinase in cerebrospinal fluid}

\author{
R. L. NEWMAN From the Department of \\ Pathology, Queen Mary's Hospital for Children, \\ Carshalton
}

One of the difficulties encountered in intravenous therapy with streptokinase as a plasminogen activator is the early development of antibodies during treatment which, in addition to neutralizing the activator, may also cause sensitivity reactions. The presence of pre-formed antibodies markedly influences the dose of activator necessary to achieve a satisfactory thrombolytic state in plasma. These factors are also of importance if intrathecal streptokinase is used to induce a fibrinolytic state in the cerebrospinal fluid (Stewart, 1964). A method has been devised, therefore, for the detection and measurement of such antibodies, by measuring their effect on streptokinase in a semi-purified system where the lysis time under controlled conditions is known. A standardized bovine fibrin clot is made incorporating a known amount of streptokinase and human plasminogen, the lysis time of which is predictable. Addition of cerebrospinal fluid to the mixture before clotting will lengthen the lysis time if antibodies to streptokinase are present. Two difficulties have to be overcome. First, there must be no streptokinase or other activator in the cerebrospinal fluid as this will alter the lysis time of the system. Secondly, the amount of plasminogen in the system might be altered by the addition of a plasminogen-rich cerebrospinal fluid. This is overcome by the enriching of the system with excess plasminogen so that the largest amount likely to be added in any cerebrospinal fluid will be insignificant. The effect of varying the plasminogen content is shown in Figure 1. It will be seen that little change in lysis time is produced by an increase in plasminogen from 1 to 2

Received for publication 13 May 1964.

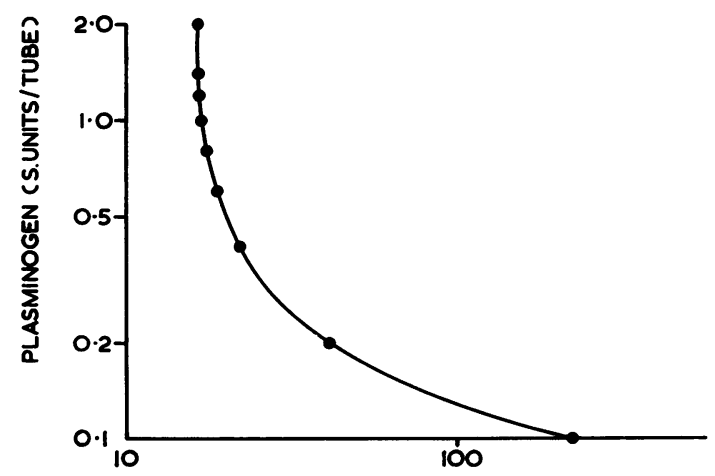

FIG. 1 . 